\title{
UNA PROPUESTA DE TRABAJOS PRÁCTICOS DE LABORATORIO QUE FAVORECE EL APRENDIZAJE DE CONCEPTOS
}

\section{A proposal for laboratory practice in favor of concept learning}

\author{
Marisol Montino ${ }^{1}$ \\ Diego Petrucci ${ }^{2}$
}

\author{
José Ernesto Ure \\ Alejandra Aleman ${ }^{4}$ \\ Silvia Margarita Pérez ${ }^{5}$
}

Resumen: Se presenta el análisis y discusión de la implementación de una modalidad de trabajo práctico de laboratorio (TPL) para el nivel universitario, desarrollada a partir de los resultados de investigaciones propias. El formato pretende que los estudiantes, a partir de tener claros los objetivos del TPL, puedan tomar decisiones que los lleven a realizar la tarea planteada. De este modo se evita que los realicen mecánicamente. La evaluación de las implementaciones muestra que los estudiantes se comprometieron con la tarea haciendo suyo el objetivo del TPL y tomando decisiones durante el desarrollo del mismo. La propuesta parece generar en los estudiantes seguridad, compromiso, actitud lúdica y participación proactiva. Por otra parte, permite establecer conexiones entre la teoría y los fenómenos dado que, en general, los estudiantes recurren inicialmente a sus ideas previas para finalmente conectar la tarea con la teoría, estableciendo relaciones que favorecen el aprendizaje de la disciplina.

Palabras clave: Didáctica. Física. Trabajo Práctico de Laboratorio. Ensino universitario.

\begin{abstract}
We present the analysis and discussion of a type of laboratory practice (TPL) for university level. The new format attempts to enable the students, starting with a clear understanding of the TPL, to take decisions which lead them to do the proposed routine. This way we try to avoid students doing the TPL mechanically. The evaluation of the implemented TPL shows that the students are committed to the task and make the objective of the TPL their own, taking decisions during the laboratory sessions. This proposal seems to generate in the students feelings of assurance, compromise, playfulness and proactive participation. The proposed format allows the students to establish relations between the theory and the phenomena. They initially resort to their previous ideas and finally make the connection between the task and the theory and so establish relations which favor the learning of the discipline.
\end{abstract}

Keywords: Science education. Physics. Laboratory practice. University level.

${ }^{1}$ Grado en Física. Profesora, Departamento de Física, Instituto de Ciencias, Universidad Nacional de General Sarmiento, Los Polvorines. Buenos Aires, Argentina.<mmontino@ungs.edu.ar>

${ }^{2}$ Grado en Física y Matemática, doctor en Didáctica de las Ciencias Experimentales. Profesor, Departamento de Didáctica de la Física, Instituto de Desarrollo Humano, Universidad Nacional de General Sarmiento, Los Polvorines. Buenos Aires, Argentina. <dpetrucc@ungs.edu.ar>

${ }^{3}$ Grado en Ciencias Físicas, doutor en Física. Profesor asociado, Departamento de Física, Instituto de Ciencias, Universidad Nacional de General Sarmiento, Los Polvorines. Buenos Aires, Argentina. <ure@ungs.edu.ar>

${ }^{4}$ Grado en Física. Profesora, Departamento de Didáctica de la Física, Instituto de Desarrollo Humano, Universidad Nacional de General Sarmiento, Los Polvorines - Buenos Aires, Argentina. <maleman@ungs.edu.ar>

${ }^{5}$ Grado en Física, master en Psicología Cognitiva y Aprendizaje. Profesora, Departamento de Didáctica de la Física, Instituto de Desarrollo Humano, Universidad Nacional de General Sarmiento, Los Polvorines. Buenos Aires, Argentina. <sperez@ungs.edu.ar>

${ }^{1}$ Instituto de Ciencias, Planta Baja - Oficina 15

J. M. Gutiérrez 1150

Los Polvorines CP 1316

Buenos Aires, Argentina 
Montino M. et al.

\section{Introducción}

En 1993 ya se planteaba que "existem poucos dados empíricos sobre os mecanismos intelectuais utilizados pelos estudantes quando são convidados a realizar ou observar e a explicar os resultados de uma determinada experiência" (VILLANI; CARVALHO, 1993, p. 75). A pesar de los años que han pasado poco se ha avanzado en la comprensión de los mecanismos intelectuales involucrados en la observación y explicación de resultados experimentales. Sin embargo, sí se ha logrado ir reconociendo la influencia que tiene la forma de presentar los trabajos prácticos de laboratorio (TPL) a los estudiantes (SERÉ; COELHO; NUNES, 2003; ANDRÉS Z.; PESA, 2004; ANDRÉS; PESA; MOREIRA, 2006).

En la bibliografía consultada no hay acuerdo respecto de qué es un trabajo práctico de laboratorio; García Sastre, Insausti y Merino (1999) realizan un interesante resumen del estado del debate sobre el tema, con argumentos a favor y en contra de los TPL. Por otro lado, Tenreiro-Vieira y Vieira (2006) presentan un resumen de las diferentes clasificaciones o tipos de TPL, cada uno con sus correspondientes objetivos. En varios trabajos se presentan diferentes objetivos y muchos de ellos son cuestionados por distintos autores con sólidos argumentos (GARCÍA SASTRE; INSAUSTI; MERINO, 1999; HODSON, 1994; PETRUCCI; URE; SALOMONE, 2006; SERÉ, 2002; WHITE, 1996).

En este trabajo se presenta el análisis de la implementación de una modalidad de TPL. Fue desarrollada a partir de criterios elaborados en base a resultados de investigaciones propias sobre el rol de los TPL de física en la formación de estudiantes universitarios (PETRUCCI; URE; SALOMONE, 2006; MONTINO; PETRUCCI; URE, 2006).

\section{Fundamentación}

Los objetivos generales de la investigación en la que se enmarca este trabajo son:

- Conocer el rol de los TPL en el aprendizaje de Física.

. Conocer la influencia de los distintos tipos de TPL en la formación de los estudiantes.

Para ello era importante saber:

- ¿Qué piensan los estudiantes de los TPL?

- ¿Qué hacen los estudiantes cuando realizan los TPL?

La investigación se efectuó con estudiantes de Física General de la Universidad Nacional de General Sarmiento situada en el segundo cordón del conurbano bonaerense. Es la primera materia de Física que cursan los estudiantes de Profesorados de Física y Matemática, Ingenierías, Ecología Urbana y Urbanismo. El programa abarca la primera parte de la mecánica de la partícula. Tiene un alto índice de recursantes (alrededor de 50\%). Las clases son teórico-prácticas e incluyen exposiciones teóricas, resolución de problemas de lápiz y papel y los TPL. Cabe aclarar que todos los estudiantes que ingresan a la Universidad tuvieron la materia Física durante la enseñanza media, si bien los contenidos trabajados incluyeron en general poca o ninguna física (por ejemplo se limitó a la enseñanza de suma de vectores o cambio de unidades). A continuación se presentan los resultados obtenidos previamente, que se resumen en el Cuadro 1. 
Una propuesta de trabajos prácticos ...

Cuadro 1. Resumen de los resultados previos de la investigación.

\begin{tabular}{|l|l|}
\hline \multicolumn{1}{|c|}{ Tienen dificultades para: } & \multicolumn{1}{c|}{ Valoran: } \\
\hline . Comprender los objetivos de los TPL. & $\begin{array}{l}\text {. Los TPL como herramienta de visualización } \\
\text { (donde "ven" los conceptos del pizarrón). } \\
\text {. Darle significado a las tareas que realizan. } \\
\text {. Desempeñarse en el contexto en que se realizan los TPL. } \\
\text { unificar y comprender lo que hicieron. }\end{array}$ \\
\hline $\begin{array}{l}\text { En general no utilizan criterios de validación internos (lógicos o disciplinares) sino por autoridad: docente, } \\
\text { libro, guía de TP o un compañero, realizando los TPL de manera mecánica. }\end{array}$
\end{tabular}

En una primera etapa se indagó sobre el rol de los TPL en los cursos de Física, mediante entrevistas tomadas a un grupo estudiantes universitarios avanzados. Los resultados (PETRUCCI; URE; SALOMONE, 2006) mostraron que los estudiantes tienen dificultades para comprender los objetivos planteados por los docentes, para darle significado a las tareas que realizan y, en general, para realizar los TPL. Por otro lado los valoran como una herramienta de visualización, donde "ven" los conceptos explicados en el pizarrón. Además, aprovechan la instancia de redacción del informe para unificar y comprender lo que hicieron en el laboratorio. El cálculo de errores les fue presentado con un grado de dificultad formal que superaba su capacidad de comprensión e indicaron que no volvieron a utilizar este procedimiento en cursos posteriores.

La segunda etapa de la investigación continuó con la observación y entrevistas posteriores a dos grupos de estudiantes durante el desarrollo de un TPL tradicional (Anexo I), de "estructura clásica" (RICHOUX; BEAUFILS, 2003) cuantitativo, en el que el sistema de estudio se presentó a los estudiantes ya modelizado e involucró mediciones y tratamientos numéricos. En esta segunda etapa se detectó que los estudiantes nunca utilizaron criterios de validación internos, es decir, recurriendo a la lógica o al saber disciplinar. Los criterios utilizados fueron de tipo externo, apelando a una autoridad: el docente, un libro, la guía de trabajos prácticos o un compañero (MONTINO; PETRUCCI; URE, 2006). Del análisis de las entrevistas se obtuvieron resultados compatibles con los anteriores: rescataron la utilidad de los TPL como instrumentos de visualización y la instancia de redacción del informe como el momento de unificar y comprender lo que hicieron; señalaron el cálculo del error como la tarea más compleja durante el TPL y la redacción del informe correspondiente. Por otro lado, los integrantes de uno de los grupos observados manifestaron sentir vergüenza de consultar al docente por "miedo" a dejar en evidencia que no sabían lo que, ellos suponían, debían saber.

\section{Diseño de modalidad de TPL}

Considerando los resultados reseñados se decidió proponer una modalidad no tradicional con las siguientes características:

- Que permita que los estudiantes se involucren con la tarea y que ésta les represente un desafío que puedan resolver experimentalmente. 
Montino M. et al.

- Que el TPL sea una secuencia de tareas integradas, que oriente a los estudiantes en el desarrollo del trabajo a realizar.

- Que el tema sea sencillo y accesible, dado que es una de las primeras veces que se enfrentan al trabajo de laboratorio.

- Que el tema sea desafiante y cuya resolución esté al alcance de los estudiantes.

En función de esas características se decidió comenzar con una pregunta para la cual los estudiantes pudieran formular una respuesta. Las respuestas son el punto de partida desde el cual cada grupo desarrolla sus diseños experimentales y decide las mediciones a realizar. El TPL consiste, precisamente, en llevar a cabo esas tareas (MONTINO; PETRUCCI; URE, 2007). En el Cuadro 2 se muestra un esquema de la modalidad propuesta.

Cuadro 2. Estructura de la modalidad de TPL propuesta.

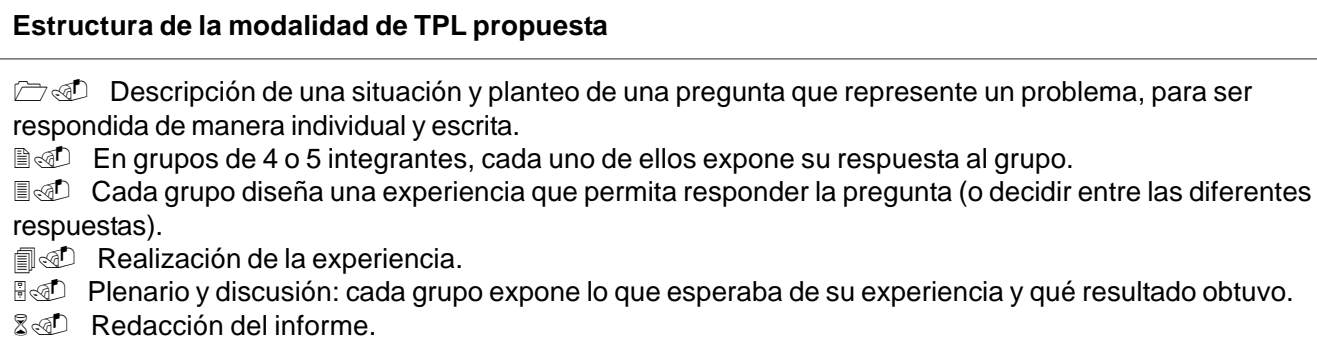

\section{Metodología para la toma y el análisis de datos}

La metodología utilizada puede ser caracterizada como exploratoria, cualitativa y sin categorías determinadas a priori por el marco teórico (GLASER; STRAUSS, 1968). Es de tipo exploratoria debido a que no se pretende confirmar o descartar hipótesis ya formuladas, sino que las mismas se van definiendo en el transcurso de la investigación (ERICKSON, 1997). Los datos con que se trabajará serán de naturaleza cualitativa (respuestas orales o escritas y registro de observaciones). Las categorías para clasificar los datos surgirán del análisis de los mismos, agrupando aquellos que tienen características similares, es decir que no se utilizarán categorías a priori.

Se diseñaron, e implementaron en varias oportunidades, tres TPL dentro de la modalidad propuesta, que se identifican con los nombres Cañoncitos, Plano inclinado y Carrito (ver Anexos 2, 3 y 4). En cinco de las implementaciones se realizaron observaciones sin registro escrito; es decir, se observó el desarrollo general de toda la comisión. Se recolectaron los informes entregados por los estudiantes. Además se realizó una observación con registro escrito del trabajo de un grupo durante la realización de uno de los TPL y entrevistas posteriores a los integrantes del grupo (con la misma modalidad y guión de entrevista que las realizadas en la etapa previa de la investigación). 
Una propuesta de trabajos prácticos ...

\section{Discusión de los datos}

\section{Resultados de las implementaciones de los TPL propuestos}

Los resultados del análisis de las observaciones, las entrevistas y los informes son:

. Se obtuvo un grado de participación y compromiso por parte de la mayoría de los estudiantes sensiblemente superior al de otras clases. Los estudiantes trabajaron animadamente y sin interrupciones y los grupos requirieron pocas intervenciones de los docentes.

- Los estudiantes comprendieron desde el inicio del TPL cuál era el objetivo del mismo. Esta afirmación se basa en dos indicadores: tomaron decisiones durante el transcurso del trabajo, en función de su objetivo y los informes reflejaron las tareas realizadas por los grupos en el transcurso del laboratorio. Éstas fueron expuestas en los plenarios finales, dónde los diferentes grupos participaron activamente.

- En las implementaciones de los TPL "Cañoncitos" y "Plano inclinado" los estudiantes no recurrieron a la teoría para contestar individualmente la pregunta inicial, pese a que era un tema ya visto en las clases previas. Es más, en el caso de los "Cañoncitos", la pregunta podía ser contestada solamente recurriendo al marco teórico (sin experimentar). Únicamente recurrieron a la teoría en el TPL "Plano inclinado" al momento de decidir cómo analizar los datos obtenidos por medición, en muchos casos por sugerencia de los docentes. Los estudiantes del grupo observado se diferenciaron de los anteriores porque, apenas contestaron la pregunta desde la intuición, comenzaron a utilizar la teoría para encontrar la respuesta, identificando las fuerzas que actuaban sobre cada cuerpo, planteando las ecuaciones y buscando despejar la aceleración.

. Durante los plenarios finales del TPL “Cañoncitos", todos los estudiantes afirmaron que ambas pelotitas llegaban juntas al suelo aunque las mediciones que habían realizado indicaban ligeras diferencias. Esto sería un indicador de que los estudiantes estarían empleando una noción de incertidumbre intrínseca a la medición, aunque no puedan explicitarlo, y aplicando criterios propios para decidir si dos valores diferentes pueden considerarse iguales.

\section{Diferencias y similitudes halladas entre modalidad tradicional y la modalidad propuesta}

Como resultado del análisis de las tres observaciones con registro escrito (dos del formato tradicional y una de la propuesta) y sus respectivas entrevistas, se encontró que (se presenta un resumen en el Cuadro 3):

. Durante los TPL con formato tradicional los grupos utilizaron siempre criterios de validación externos al momento de tomar decisiones (MONTINO; PETRUCCI; URE, 2006). En cambio, en el grupo que realizó el TPL con la modalidad propuesta las decisiones fueron resultado de debates con argumentos, que respondían a la lógica o el saber disciplinar.

. En relación con el objetivo de la tarea, que en los tres casos era calcular la aceleración del sistema, los grupos que realizaron el TPL con formato tradicional no lograron describirlo durante la entrevista: 
Montino M. et al.

[...] Bueno, y eso nos servía... para sacar la fuerza de rozamiento que... era nuestro objetivo, creo que era lo mas importante... la fuerza de rozamiento y otro era sacar la gravedad... y conseguir... la gravedad y compararla con, no se. (Entrevista Obs. 1 - estudiante 1)

A ver si existía fuerza de rozamiento entre el carrito y la pista... bah, eso es lo que yo planteé como objetivo en mi informe. (Entrevista estudiante 2)

$[. .$.$] y ver digamos... lo aprendido en clase, con respecto al cálculo de error...y$ sacar nuestras propias conclusiones a través de... no que te digan "esto es asi" sino ver que sucede, y de abi sacar tus conclusiones y si es o no es lo que quería llegar el profesor. (Entrevista estudiante 5)

. Mientras que los entrevistados que realizaron el TPL con la modalidad propuesta contestaron sin dificultades cuál era el objetivo de la tarea:

El objetivo era demostrar que al aumentar la masa de un objeto que... está sujeto a otro, si existe una aceleración que no se duplica. (Entrevista estudiante 7)

Demostrar que la aceleración, o sea que la masa, que al duplicarse la masa la aceleración no se duplicaba. (Entrevista estudiante 8)

Demostrar, si se duplicaba o no la aceleración. (Entrevista estudiante 9)

. Durante la entrevista se les preguntó a los estudiantes: "¿Me podrías contar el TPL como se lo contarias a un compañero que no vino y le tenés que explicar qué bicieron?". Los estudiantes de los grupos que realizaron el TPL con formato tradicional respondieron describiendo el dispositivo experimental (muy vagamente) apoyándose mucho en el lenguaje gestual. Por el contrario, los estudiantes del TPL propuesto hicieron referencia al objetivo y no al dispositivo o a los procedimientos.

Cuadro 3. Diferencias halladas entre modalidad tradicional y la modalidad.

\begin{tabular}{|l|l|}
\hline \multicolumn{1}{|c|}{ TPL Tradicional } & \multicolumn{1}{|c|}{ TPL propuesto } \\
\hline \multicolumn{2}{|c|}{ Validación } \\
\hline $\begin{array}{l}\text { Los estudiantes utilizaron siempre criterios de } \\
\text { validación externos al momento de tomar decisiones. }\end{array}$ & $\begin{array}{l}\text { Las decisiones que tomaron los estudiantes fueron } \\
\text { resultado de debates con argumentos. }\end{array}$ \\
\hline \multicolumn{2}{|c|}{ Objescripción de la experiencia } \\
\hline \begin{tabular}{l} 
Los estudiantes no lograron describirlo. \\
\hline $\begin{array}{l}\text { Los estudiantes describieron el dispositivo } \\
\text { experimental apoyándose en el lenguaje gestual. }\end{array}$
\end{tabular} \\
\hline
\end{tabular}


Una propuesta de trabajos prácticos ...

\section{Conclusiones}

Como se muestra en los resultados de las etapas previas de la investigación, el formato tradicional de TPL no parece favorecer los aprendizajes de conceptos, de procedimientos ni de actitudes. A continuación se delinean las ventajas halladas en las implementaciones de los TPL propuestos:

- Desde una perspectiva actitudinal, parecen generar en los estudiantes seguridad, compromiso, actitud lúdica y participación proactiva frente al problema propuesto; a diferencia del formato tradicional donde se encontraba frustración, desgano, indiferencia y actitud reactiva. El clima de la clase fue una pauta de que los estudiantes le dieron sentido a las actividades en el momento de desarrollarlas. Es posible que encontrarle sentido a la actividad haya contribuido al compromiso generando un convencimiento de la tarea y de sus resultados.

. Desde una perspectiva conceptual, permiten a los estudiantes establecer conexiones entre la teoría y los fenómenos, ya que el problema les resulta accesible y no arbitrario. Los estudiantes lo sienten como un problema cuya resolución está a su alcance y por lo tanto los criterios de validación se vuelven internos. En general recurren inicialmente a sus ideas previas para finalmente conectar la tarea con la teoría, estableciendo relaciones que favorecen el aprendizaje de la disciplina.

- Desde una perspectiva procedimental, los estudiantes tuvieron iniciativas y tomaron decisiones durante el desarrollo de los TPL. Se atribuye este resultado a la estructura de los TPL, que permite que los estudiantes organicen su trabajo en función a las decisiones que toman para llegar a un resultado que les permita responder la pregunta, como por ejemplo decidir el número de repeticiones de la experiencia. Esto está en concordancia con "a repetição dos experimentos ofereceu aquele espaço de reflexão necessário para tomar seriamente em consideração os re-sultados experimentais e encontrar novos elementos interessantes" (VILLANI; CARVALHO, 1993, p. 84).

Una pregunta inicial adecuada hace que los estudiantes se apropien del problema, se interesen en resolverlo y los motiva a encontrar una respuesta o a verificar si su respuesta inicial era correcta o no. Según los datos recabados, los resultados de un experimento no necesariamente tenían que ser sorprendentes para motivar a los estudiantes, bastaba con que se comprometieran a responder la pregunta y realizaran las tareas planificadas por ellos mismos, sin intentar sólo complacer al docente.

La modalidad de TPL propuesta, al permitir la comprensión de su objetivo desde el comienzo de la práctica, contribuye a que los estudiantes vean al dispositivo experimental como un medio y no como un fin en sí mismo; a diferencia del TPL tradicional donde el uso intensivo del lenguaje gestual para describirlo puede atribuirse, tal vez, a un recuerdo de la experiencia más ligado a representaciones visuales que conceptuales.

Desde la perspectiva de su aprendizaje, a los estudiantes aún no les resulta sencillo utilizar un referencial teórico disciplinar en el laboratorio y ello explica en parte varias de las dificultades que suelen encontrar durante el trabajo: la observación de experimentos, identificar las variables relevantes o privilegiar los eventos más importantes (VILLANI; CARVALHO, 1993). En este contexto, los estudiantes difícilmente se proponen - y rara vez logran conectar la situación experimental planteada en el laboratorio con el tema visto en las clases teóricas, una muestra de ello es que al comienzo de los TPL propuestos no recurren a la teoría 
Montino M. et al.

para responder las preguntas disparadoras. La conexión entre el mundo de los hechos y el mundo de las ideas se hace utilizando modelos (COLINVAUX; BARROS, 2002). En los TPL propuestos los estudiantes responden inicialmente a una pregunta que se refiere al mundo de los hechos desde las ideas en un contexto cotidiano, pero finalmente todos recurrieron a la teoría en algún momento del desarrollo del TPL, modelizando las situaciones.

En estas implementaciones no se trabajó con propagación de errores, aunque en el plenario final de cada TPL se discutió sobre el significado de la incerteza en las mediciones. Este tema podría profundizarse en las materias siguientes, a medida que los estudiantes vayan teniendo más experiencia en el laboratorio, en el conocimiento del marco teórico disciplinar y más en general, comprendiendo la lógica de la disciplina. De este modo, el tema se introduce gradualmente, evitando que se transforme en un obstáculo.

Sin embargo y más allá de las virtudes planteadas, estas innovaciones en los prácticos de laboratorio no han incidido en los porcentajes de aprobación. Esto puede deberse a que la realización de los TPL y la entrega de los informes correspondientes forman parte de la evaluación que se realiza en la materia, pero no tienen incidencia en la acreditación de la misma. Pero esto no significa necesariamente que no se haya incidido en el nivel de comprensión sobre determinados temas. Por otra parte, queda pendiente analizar cómo la modalidad de TPL propuesta cambia la motivación hacia la materia, "un profesor puede facilitar la motivación de sus alumnos [...] haciendo más probable el éxito al adecuar las tareas a las verdaderas capacidades y disposiciones de sus alumnos" (POZO; GOMEZ CRESPO, 2000, p. 50).

El laboratorio es un espacio complejo donde, para los estudiantes, entran en juego muchos aspectos nuevos, el contexto de trabajo, los problemas presentados en forma diferente a la habitual de las guías, la manipulación de instrumental, etc. La propuesta de los TPL presentados simplifica algunos de estos aspectos, generando las condiciones de accesibilidad y confianza para iniciarse en ese nuevo espacio.

\section{Referencias}

ANDRÉS, M. M.; PESA, M. A. Conceptos-en-acción y teoremas-en-acción en un trabajo de laboratorio de física. Revista Brasileira de Pesquisa em Educação em Ciências, Belo Horizonte, v. 4, n. 1, p. 59-75, 2004.

.; __..; MOREIRA, M. A. El trabajo de laboratorio en cursos de física desde la teoría de campos conceptuales. Ciência \& Educação, Bauru, v. 12, n. 2, p. 129-142, 2006.

COLINVAUX, D.; BARROS, S. O papel da modelagem no laboratório didático de física: o que há para se aprender? In: ENCONTRO DE PESQUISA EM ENSINO DE FÍSICA, 8., 2002, São Paulo. Atas... São Paulo: SBF, 2002. 1 cd-rom.

ERICKSON, F. Métodos cualitativos de investigación sobre la enseñanza. In: WITTROCK, M. (Org.). La investigación de la enseñanza, II. Barcelona: Paidós Educador, 1997. p. 195-301. 
Una propuesta de trabajos prácticos ...

GARCÍA SASTRE, P.; INSAUSTI, M. J.; MERINO, M. Propuesta de un modelo de trabajos prácticos de física en el nivel universitario. Enseñanza de las Ciencias, Barcelona, v. 17 , n. 3, p. 533-542, 1999.

GLASER, B.; STRAUSS, A. The discovery of grounded theory: strategies for qualitative research. London: Weidenfeld \& Nicholson, 1968.

HODSON, D. Hacia un enfoque más crítico del trabajo de laboratorio. Enseñanza de las Ciencias, Barcelona, v. 12, n. 3, p. 299-313, 1994.

MONTINO, M.; PETRUCCI, D.; URE, J. E. ¿Magia o física? Los estudiantes universitarios y los trabajos prácticos de laboratorio. In: SIMPOSIO DE INVESTIGACIÓN EN EDUCACIÓN EN FÍSICA, 8., 2006, Gualeguaychú. Memorias... Gualeguaychú: APFA, 2006. p. 367-375. 1 cd-rom.

$\therefore$

Propuesta de modalidad de trabajo práctico de laboratorio para

el nivel universitario básico. In: REUNIÓN DE ENSEÑANZA DE LA FÍSICA, 15., 2007, Merlo. Memorias... Merlo: APFA, 2007. 1 cd-rom.

PETRUCCI, D.; URE, J. E. La visión de estudiantes universitarios sobre los trabajos prácticos de laboratorio de física. In: ENCONTRO DE PESQUISA EM ENSINO DE FÍSICA, 10., 2006, Londrina. Memórias... Londrina: SBF, 2007. 1 cd-rom.

.;___ _ SALOMONE, H. D. Cómo ven a los trabajos prácticos de laboratorio de física los estudiantes universitarios. Revista de Enseñanza de la Física, Rosario, v. 19, n. 1, p. 7-20, 2006.

POZO, J. I.; GÓMEZ CRESPO, M. A. Aprender y enseñar ciencia. 2. ed. Madrid: Morata, 2000.

RICHOUX, H.; BEAUFILS, D. La planificación de las actividades de los estudiantes en los trabajos prácticos de física: análisis de prácticas de profesores. Enseñanza de las Ciencias, Barcelona, v. 21, n. 1, p. 95-106, 2003.

SERÉ, M. G. La enseñanza en el laboratorio. ¿Qué podemos aprender en términos de conocimiento práctico y de actitudes hacia la ciencia? Enseñanza de las Ciencias, Barcelona, v. 20, n. 3, p. 357-368, 2002.

SERÉ, M.-G. ; COELHO, S. M.; NUNES, A. D. O papel da experimentação no ensino da física. Caderno Brasileiro de Ensino de Física, Florianópolis, v. 20, n. 1, p. 30-42, 2003.

TENREIRO-VIEIRA, C.; VIEIRA, R. M. Diseño y validación de actividades de laboratorio para promover el pensamiento crítico de los alumnos. Revista Eureka sobre Enseñanza y Divulgación de las Ciencias, Cadiz, v. 3, n. 3, p. 452-466, 2006.

VILLANI, A.; CARVALHO, L. Representações mentais experimentos cualitativos. Revista Brasileira de Ensino de Física, São Paulo, v. 15, n. 1, p. 74-89, 1993.

WHITE, R. T. The link between the laboratory and learning. International Journal of Science Education, Colchester, v. 18, n. 7, p. 761-774, 1996. 
Montino M. et al.

\section{Anexo I - TPL “Carrito 1" - Formato tradicional}

Se presentaba a los estudiantes el siguiente dispositivo experimental (ver Figura 1) conformado por: un carrito, una pista, una polea, pesas, un hilo y dos photogates. Por otra parte contaban con cinta métrica y una balanza. Se les pedía calcular la aceleración de los cuerpos.

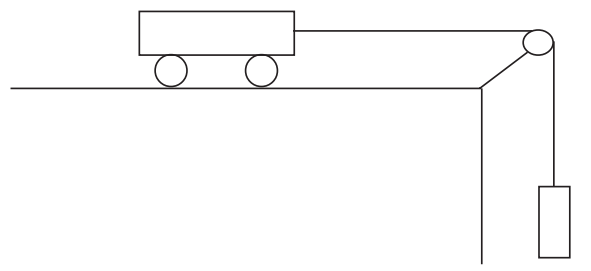

Figura 1. Esquema del dispositivo experimental

\section{Anexo II - TPL "Cañoncitos"}

Desde una mesa se lanzan simultáneamente dos bolitas con distinta velocidad horizontal.

1- En forma individual, responda por escrito ¿Cuál llega primero al suelo? ¿Por qué?

2- En grupos de 4 o 5 integrantes.

Cada integrante cuenta su respuesta al grupo.

3- Entre todos diseñan y realizan una experiencia.

4- Plenario final y discusión de los resultados.

Cada grupo cuenta lo que esperaba de su experiencia y que resultado obtuvo.

5- Informe en casa.

a- pregunta inicial y discusión grupal y plenaria.

b- la experiencia realizada y elementos utilizados.

c- resultados obtenidos.

d- conclusiones.

\section{Anexo III - TPL “Plano Inclinado"}

1- Familiarizarse con el equipo, jugar un rato, y preguntar para saber sobre el funcionamiento de los distintos componentes.

2- Encuesta individual.

Si inclinamos levemente la pista y dejamos caer el carrito desde el extremo a mayor altura. ¿Qué tipo de movimiento realiza el carrito (MRU o MRUV)?

Ciência \&̊ Educacão, v. 17, n. 4, p. 823-833, 2011 
3- En grupos de 4 o 5 integrantes.

Comentar lo que cada uno respondió a la pregunta inicial.

¿Qué mediciones se pueden hacer para decidir cual es el tipo de movimiento? Justificar las decisiones tomadas.

4- Realizar las mediciones.

5- Preparar una exposición del trabajo realizado.

6- Puesta en común. Cada grupo cuenta las mediciones que hizo, por qué las propuso y qué resultados obtuvo.

7- Plenario final y discusión de resultados.

\section{Anexo IV - TPL "Carrito 2"}

La empresa "Tecnabo" fabrica tecnología de punta, tiene un problema y ha pedido asesoramiento profesional a la Universidad. Los operarios bajan los componentes fabricados por una ventana hasta el camión con un sofisticado sistema:

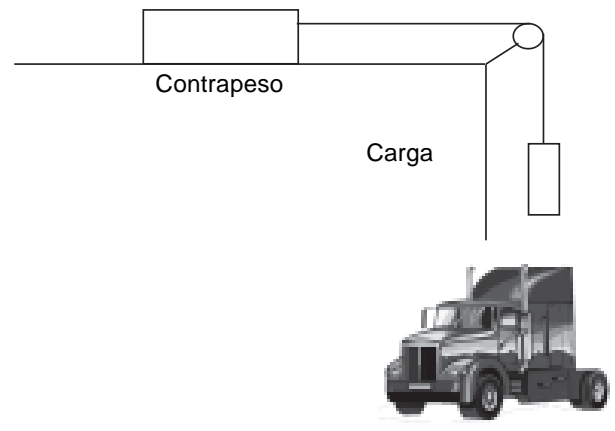
Necesitan aumentar la eficiencia, para lo cual están pensando en duplicar la carga bajada cada vez, pero un operario que estaba cursando Física General sospechó que ello podría ser riesgoso para los delicados componentes, porque aumentaría la aceleración. Hizo algunas cuentas y concluyó que sería peligroso que la aceleración aumente al doble. El asesoramiento consiste en analizar si pueden o no duplicar la carga. Los docentes de la materia hemos elaborado el siguiente trabajo práctico:

Dado el siguiente dispositivo:

1- En forma individual, responder por escrito:

Si duplico la masa $\mathrm{m}_{2}$ ¿se duplica la aceleración del sistema? Justifique

2- En grupos de 4 o 5 integrantes, cada integrante cuenta su respuesta al grupo.

3- Entre todos diseñan y realizan una experiencia que permita contestar la pregunta inicial.

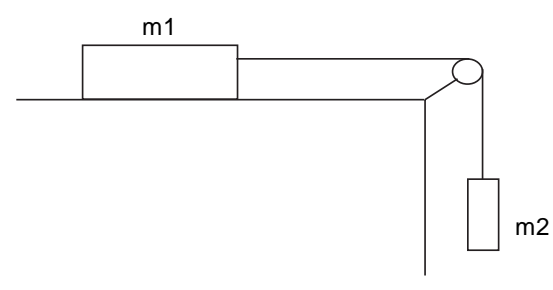

4- Plenario final y discusión de los resultados, cada grupo cuenta lo que esperaba de su experiencia y qué resultado obtuvo.

5- Elaborar un informe por grupo a entregar en una semana. 